\title{
Correction to: Characterization of the virulence of Pseudomonas aeruginosa strains causing ventilator-associated pneumonia
}

\author{
Beatriz Alonso ${ }^{1,2^{*}}$, Laia Fernández-Barat ${ }^{3,4}$, Enea Gino Di Domenico ${ }^{5}$, Mercedes Marín ${ }^{1}$, Emilia Cercenado ${ }^{1}$, \\ Irene Merino ${ }^{6,7,8,9,10}$, Manuela de Pablos ${ }^{11}$, Patricia Muñoz ${ }^{1,2,12,13}$ and María Guembe ${ }^{1,2}$
}

\section{Correction to: BMC Infect Dis (2020) 20:909 \\ https://doi.org/10.1186/s12879-020-05534-1}

Following publication of the original article [1], the authors identified an error in the caption of Figs. 1 and 2: these should be inverted.

The correct captions are given below.

The correct caption for Fig. 1 is: "Classification of pyoverdine and pyocyanin producer by qualitative observational method. a, pyoverdine producer strain; b, pyocyanin producer strain"

The correct caption for Fig. 2 is: "Survival curves for Galleria mellonella larvae after infection with Pseudomonas aeruginosa strains isolated from patients with VAP (VAP $P$. aeruginosa, $n=8$ ) and from patients with other infections (non-VAP $P$. aeruginosa, $n=8$ ). PBS was used as a positive control for larvae survival. "Statistically differences were found between VAP and nonVAP $P$. aeruginosa $(p<0.001)$ using the log-rank test".

The original article [1] has been updated.

\section{Author details}

'Department of Clinical Microbiology and Infectious Diseases, Hospital General Universitario Gregorio Marañón, Madrid, Spain. ${ }^{2}$ Instituto de Investigación Sanitaria Gregorio Marañón, Madrid, Spain. ${ }^{3}$ Centro de Investigación Biomedica En Red-Enfermedades Respiratorias (CibeRes, CB06/
06/0028) and Institut d'Investigacions Biomèdiques August Pi i Sunyer (IDIBAPS), Barcelona, Spain. ${ }^{4}$ Center for Biomedical Research CELLEX, School of Medicine, University of Barcelona, Barcelona, Spain. ${ }^{5}$ San Gallicano Dermatological Institute IRCCS, 00144 Rome, Italy. ${ }^{6}$ Servicio de Microbiología, Hospital Universitario Ramón y Cajal and Instituto Ramón y Cajal de Investigación Sanitaria (IRYCIS), Madrid, Spain. ${ }^{7}$ Red Española de Investigación en Patología Infecciosa (REIPI), Madrid, Spain. ${ }^{8}$ Group For Biomedical Research in Sepsis (BioSepsis) Hospital Clínico Universitario de Valladolid, Valladolid, Spain. ${ }^{9}$ Centro de Investigación Biomedica En Red - Enfermedades Respiratorias (CibeRes, CB06/06/0028), Barcelona, Spain. ${ }^{10}$ National Health System, SACYL/IECSCYL, Valladolid, Spain. ${ }^{11}$ Servicio de Microbiología y Parasitología Hospital Universitario La Paz, Madrid, Spain. ${ }^{12} \mathrm{CIBER}$

Enfermedades Respiratorias-CIBERES (CB06/06/0058), Madrid, Spain.

${ }^{13}$ Medicine Department, School of Medicine, Universidad Complutense de Madrid, Madrid, Spain.

Published online: 11 December 2020

Reference

1. Alonso B, et al. Characterization of the virulence of Pseudomonas aeruginosa strains causing ventilator-associated pneumonia. BMC Infect Dis. 2020;20:909 https://doi.org/10.1186/s12879-020-05534-1.

The original article can be found online at https://doi.org/10.1186/s12879 020-05534-1.

* Correspondence: beaafm@hotmail.com

'Department of Clinical Microbiology and Infectious Diseases, Hospital General Universitario Gregorio Marañón, Madrid, Spain

${ }^{2}$ Instituto de Investigación Sanitaria Gregorio Marañón, Madrid, Spain

Full list of author information is available at the end of the article

C The Author(s). 2020 Open Access This article is licensed under a Creative Commons Attribution 4.0 International License, which permits use, sharing, adaptation, distribution and reproduction in any medium or format, as long as you give appropriate credit to the original author(s) and the source, provide a link to the Creative Commons licence, and indicate if changes were made. The images or other third party material in this article are included in the article's Creative Commons licence, unless indicated otherwise in a credit line to the material. If material is not included in the article's Creative Commons licence and your intended use is not permitted by statutory regulation or exceeds the permitted use, you will need to obtain permission directly from the copyright holder. To view a copy of this licence, visit http://creativecommons.org/licenses/by/4.0/. The Creative Commons Public Domain Dedication waiver (http://creativecommons.org/publicdomain/zero/1.0/) applies to the data made available in this article, unless otherwise stated in a credit line to the data. 